\title{
Cutaneous sporotrichosis with dermoscopic features: A case report
}

\section{Emine Müge Acar ${ }^{1 *}$, Ümran Günay ${ }^{2}$, Ömer Faruk Elmas³ ${ }^{3}$, Serpil Çorum Şirin $^{4}$, Asuman Kilitci $^{5}$}

\begin{abstract}
Objective: Sporotrichosis is a fungal skin infection caused by Sporothrix schenckii. We report a 70- year-old female presenting with an erythematous nodule on her right forefinger after a rose thorn prick.

Material and Methods: The nodule was histopathologically diagnosed as sporotrichosis. Dermoscopy of the nodule revealed structureless white areas with a lobular arrangement, prominent scaling, blood spots, and polymorphous vessels including coiled, punctate and looped vessels. Dermoscopy has opened a new horizon in the diagnosis of skin infections in recent years.

Conclusion: The dermoscopic features of sporotrichosis have not yet been reported as far as we are aware. We believe that coexistence of the above features, which could be thought to be nonspecific when seen separately, may be of diagnostic significance and a helpful tool in the diagnosis of cutaneous sporotrichosis.
\end{abstract}

Keywords: cutaneous sporotrichosis, Sporothrix schenckii, fungal, dermoscopy, histopathology

\section{Introduction}

Sporotrichosis is a fungal skin infection caused by the dimorphic fungus Sporothrix schenckii. The gold standard for the diagnosis is still the isolation and identification of the Sporothrix species. Dermoscopy is used as a diagnostic tool in many skin disorders and has opened a new horizon in the diagnosis of skin infections in recent years. The dermoscopic features of sporotrichosis have not previously been reported.

\section{Case}

A 70-year-old female presented with an erythematous, painless nodule on her right forefinger. The patient's history revealed that she had been pricked by a rose thorn while gardening and that the lesion had developed afterwards about 7 months ago.

The nodule had gradually enlarged during this period. Physical examination revealed an erythematous, scaly nodule measuring about $1 \times 1 \mathrm{~cm}$ (Figure 1).

Mild pruritus was present. Dermoscopy showed numerous structureless white areas in a lobular arrangement, prominent scaling, blood spots, and polymorphous vessels including coiled, punctate and looped vessels (Figure 2).
Histopathological examination of the skin biopsy revealed the hyperkeratosis, parakeratosis, epidermal hyperplasia, neutrophilic microabscess formations, eosinophilic yeastlike organisms with surrounding of eosinophilic material (PAS+), mixed type inflammatory cells infiltrate the overlying keratin layer, epidermis and dermis (Figure 3).

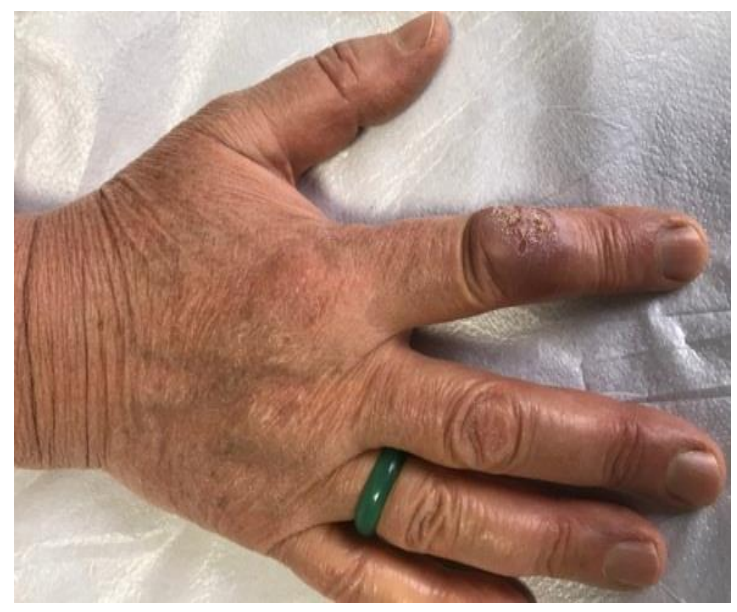

Figure 1: Erythematous nodule on the right forefinger 


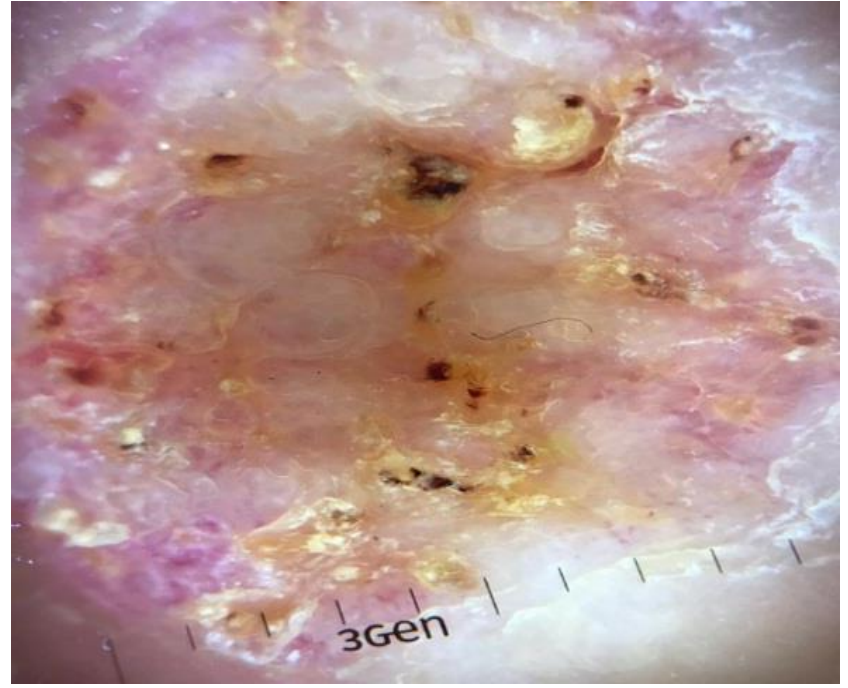

Figure 2: Numerous white structureless areas in lobular arrangement, prominent scale, blood spots, polymorphous vessels including coiled, dotted and looped vessels on dermoscopical examination
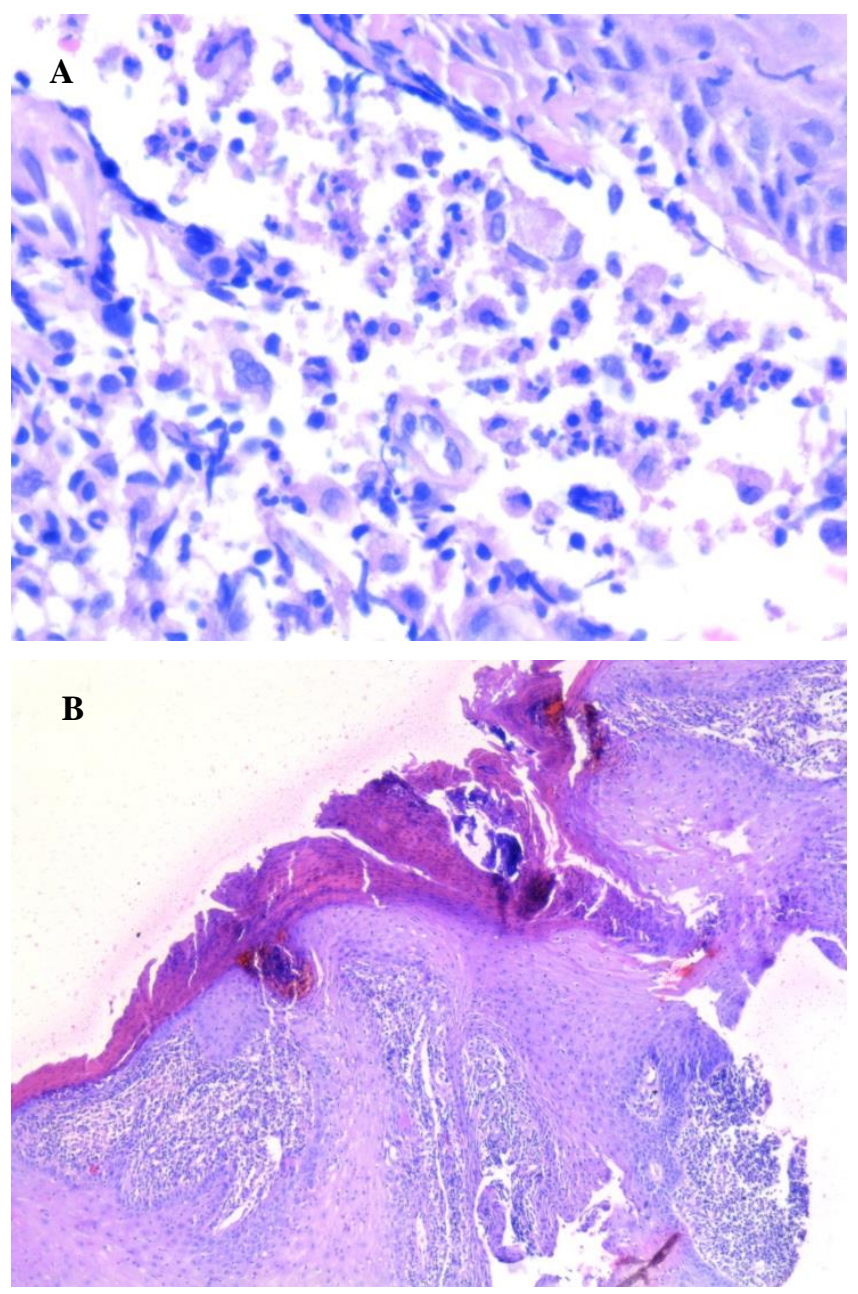

Figure 3A, 3B: Eosinophilic yeast-like organisms with surrounding of eosinophilic material; Hyperkeratosis, parakeratosis, epidermal hyperplasia, neutrophilic microabscess formations, mixed type inflammatory cells (H\&E, x400; H\&E, x100).

\section{Discussion}

Sporotrichosis (rose gardener's disease) caused by Sporothrix schenckii is the most common subcutaneous mycosis. The disease can present in the lymphocutaneous, fixed cutaneous or disseminated cutaneous form $(1,2)$. The infection usually results from the inoculation of the agents on the skin or mucosa following trauma with contaminated plant materials, leading to an increased risk in farmers, gardeners, florists and foresters (2).

Lymphocutaneous sporotrichosis is the most frequent form and accounts for about $75 \%$ of the cases (2). Our diagnosis in this case was lymphocutaneous sporotrichosis as well. The initial lesion appears as a papulonodule in this form. The nodules can then ulcerate and produce a purulent discharge. Cutaneous sporotrichosis may also present as large ulcers with well-defined borders, and papulopustular, vegetative, infiltrative and crusty lesions (3). The differential diagnosis includes leishmaniasis, cat-scratch disease, cutaneous nocardiosis, chromomycosis, syphilis, granuloma annulare, and pyoderma gangrenosum.

The gold standard for the diagnosis of sporotrichosis is the isolation and the identification of the Sporothrix species from clinical samples. Histopathology, serology and molecular studies (PCR) are the other current diagnostic methods (4). Dermoscopy is a non-invasive diagnostic tool that is mainly used for evaluating pigmented skin lesions. It is now also widely used for the diagnosis of various inflammatory and non-inflammatory disorders of the skin.

Dermoscopy of skin infections and infestations, i.e., "entodermoscopy", has opened a new horizon in the diagnosis of these disorders as a fast and practical method in recent years. There is increasing evidence showing dermoscopy to be helpful in facilitating clinical recognition, confirming the diagnosis, making the differential diagnosis, and even monitoring the treatment response (5).

Specific dermoscopic criteria have been described for several infectious diseases including lupus vulgaris, leishmaniasis, scabies, pediculosis, HPV infections, molluscum contagiosum, and tick bites (6). The dermoscopic features of sporotrichosis have not previously been reported, due to literature search. We dermoscopicallydetected numerous structureless white areas in a lobular arrangement corresponding to hyperkeratosis and acanthosis of the epidermis with prominent scaling correlating with parakeratosis. A polymorphous vascular pattern, composed of two or more different vascular patterns, can be a dermoscopic sign for malignant skin lesions and especially melanoma (7). It can also be seen in benign skin lesions such as eccrine poroma and cutaneous leishmaniasis $(8,9)$. We observed polymorphous vessels including coiled, punctate and looped vessels in our case, indicating that cutaneous sporotrichosis is another skin disease that can display a polymorphous vascular pattern. Observation of blood spots, as observed in our case, is another dermoscopic finding and can be associated with the itching and scratching. 


\section{Conclusion}

Some dermoscopic features can be highly specific to the disease as in the example of scabies, which shows a "jet with contrail" appearance, while some dermoscopic signs can be seen in multiple disorders and are therefore considered 'nonspecific' (6). However, a nonspecific feature can also help in the diagnosis and differentiation of a disorder in the presence of other dermoscopic criteria. Although we cannot say any of the dermoscopic features we observed was particularly specific for cutaneous sporotrichosis, the lobular arrangement of structureless white areas, and the presence of prominent scaling, blood spots and a polymorphous vascular pattern indicated that sporotrichosis displays a distinct dermoscopic pattern, which needs to be confirmed on larger series. We believe that dermoscopy could become a helpful non-invasive tool for the diagnosis of cutaneous sporotrichosis.

Acknowledgement: This article has been presented as an poster presentation in part at the IDEA Congress at Ankara, held at March 8-11, 2018.

Conflict of Interest: The authors declare no potential conflicts of interest with respect to the research, authorship, and/or publication of this article.

Author's Contributions: EMA, ÜG, ÖFE, SÇŞ, AK; Research concept and design, Patient examinations, Research the literature, preparation of the article. AK; Pathological examinations, Revision of the article.

\section{References}

1. Larson KN, Pandey S, Hoover W, Sun NZ. Sporotrichosis in the nailan unusual location and presentation. JAAD Case Rep. 2017;4:47-9.

2. Mahlberg MJ, Patel R, Rosenman K, Cheung W, Wang N, Sanchez M. Fixed cutaneous sporotrichosis. Dermatol Online J. 2009;15:5.

3. Orofino-Costa R, Macedo PM, Rodrigues AM, Bernardes-Engemann AR. Sporotrichosis: an update on epidemiology, etiopathogenesis, laboratory and clinical therapeutics. An Bras Dermatol. 2017;92:606-20.

4. de Lima Barros MB, de Almeida Paes, de Oliveira Schubach A. Sporothrix schenckii and Sporotrichosis. Clin Microbiol Rev. 2011;24:633-54.

5. Zalaudek I, Giacomel J, Cabo H, Di Stefani A, Ferrara G, HofmannWellenhof R. Entodermoscopy: a new tool for diagnosing skin infections and infestations. Dermatology. 2008;216:14-23.

6. Lallas A, Giacomel J, Argenziano G, García-García B, GonzálezFernández D, Zalaudek I, Dermoscopy in general dermatology: practical tips for the clinician. Br J Dermatol. 2014; 170:514-26.

7. Ayhan E, Ucmak D, Akkurt Z. Vascular structures in dermoscopy. An Bras Dermatol. 2015;90:545-53.

8. Ayhan E, Ucmak D, Baykara SN, Akkurt ZM, Arica M. Clinical and dermoscopic evaluation of cutaneous leishmaniasis. Int J Dermatol. 2015;54:193-201.

9. Ferrari A, Buccini P, Silipo V, De Simone P, Mariani G, Marenda S, Eccrine poroma: a clinical-dermoscopic study of seven cases. Acta Derm Venereol. 2009;89:160-4. 